\title{
Schori, Kurt (2020). Glaubensüberlieferungen verstehen. Eine Einführung in den Fachbereich der Religionen und in die Didaktik der heiligen Schriften. Stuttgart: Kohlhammer. ISBN 978-3-I 7-037687-8. 250 Seiten.
}

\section{Fatima Çaviş}

Kirchliche Pädagogische Hochschule Wien/Krems (fatima.cavis@kphvie.ac.at)

Das in vielen gegenwärtigen Religionen oft zerklüftet wirkende Verhältnis zwischen Glaubensaussagen und aktuellen Lebenswirklichkeit(en) der (gläubigen) Menschen auf der einen Seite; die in säkularisierten gesellschaftlichen Kontexten immer stärkere Verdrängung der Religion(en) auf den privaten Raum auf der anderen Seite - in diesem Spannungsraum bewegt sich der Beitrag des christlichen Religionspädagogen Kurt Schori.

Auf der Metaebene stellt der Autor die Frage nach der Verortung der Religion(en) und des Religiösen sowie der heiligen Schriften in einer säkularisierten Welt. Damit verbunden ist die ,Rechtfertigung' der Existenz von Religion(en) sowie des Glaubens durch die Betonung ihrer Verknüpfung mit den existenziellen Fragen und ihrer individuellen insbesondere aber sozialen Relevanz.

Dies verdeutlicht Schori im ersten Kapitel, das er gänzlich den „Glaubensfragen“ widmet. Er weist darauf hin, dass eine gesellschaftliche Tendenz bestehe, in der Denk- und Sprachkultur zwischen „,alltäglichen sowie religiösen Ritualen und Glaubenselementen“ (17) strikt zu unterscheiden, wobei die Religion bzw. das Religiöse oft u. a. als etwas, das mit Verstand nicht begreifbar und somit unbegründbar rezipiert wird (23-25). Schori greift diese Problematik kritisch auf und zielt mit seinem Beitrag darauf ab, den Menschen, die sich aus persönlichem Interesse oder beruflichen Gründen (Laien, (Religions-)Lehrer*innen etc.) mit Glaubensfragen und Glaubensüberlieferungen auseinandersetzen, sowohl in sozialen Situationen als auch in der konkreten Unterrichtspraxis in der Schule „eine Orientierung [zu] bieten, um sich in komplexen Situationen, in welchen Fragen des Glaubens eine Rolle spielen, zurecht zu finden und handlungsfähig zu sein“ (15f.). In diesem Rahmen präsentiert der Autor auch sein mehrschrittiges „Verfahren“ (5, 139-146) zur Lektüre und Didaktik von heiligen Schriften, das er in der Lehrer*innenausbildung an der Pädagogischen Hochschule Bern entwickelt hat. Der Autor verspricht mit diesem Verfahren vor allem auch den Leser*innen ohne besondere exegetische Fachkenntnisse einen Zugang zu den heiligen Texten zu ermöglichen, damit sie „die Relevanz solcher Texte [...] erkennen bzw. [...] überprüfen“ (5f.).

Die Monografie besteht insgesamt aus zwei Teilen. Unter dem Leitspruch „Glaubensäußerungen bzw. Glaubensüberzeugungen beziehen sich auf Lebens-/Erfahrungswirklichkeit(en)“, der die gesamte Monografie durchzieht, greift der Autor im ersten Teil einige komplexe glaubensbezogene Kernthemen auf, reflektiert diese aus fachwissenschaftlich-theologischer Perspektive für ein besseres Verständnis sowie hinsichtlich ihrer Verknüpfung mit den Erfahrungs- bzw. Lebenswirklichkeiten und stellt, wo möglich, Zusammenhänge zu anderen religiösen Traditionen (Judentum, Islam, Buddhismus etc.) her. Die Themen umfassen u. a. die Gottesfrage in Verbindung zum „Selbst- und Weltverständnis“ (25), der Umgang mit der Schuldfrage in Verbindung mit Gewalt, Rechtsverachtung in Zusammenhang mit dem Kernthema Messianismus, die Rolle und Bedeutung von Bekenntnissen und Gottesdiensten im Selbstverständnis und Weltverhältnis von Menschen. Ferner finden sich im ersten Teil einige Grundinformationen zu den heiligen Schriften, zur hermeneutischen Annäherung bzw. zum Textverständnis.

Der zweite Teil widmet sich dem Verständnis sowie der religionsdidaktischen Aufbereitung von heiligen Schriften der sogenannten „vorderorientalischen Religionen“ (134) für den Klassenunterricht. Dabei werden exemplarisch Texte aus der Tora, der Bibel und ein Text aus dem Koran behandelt. Schori betrachtet die heiligen Schriften als "Glaubenszeugnisse“ (138), die „Reaktionen auf Erfahrungswirklichkeit“ (140) darstellen. In seiner Textarbeit geht es darum zu zeigen, „dass sich die in ihnen vorausgesetzten oder explizit geäußerten Glaubensaussagen auf Wirklichkeit beziehen lassen“ (138). Dies setzt voraus, dass die Texte für die gegenwärtigen Leser*innen „verstehbar, beurteilbar und überprüfbar werden“ (138). Bei dem Ansatz wird ein dialogischer Zugang angestrebt, der den Leser*innen ermöglicht, mit ihren individuellen „Erzählungen“ (138) an der im Text implizierten „Erfahrungswirklichkeit“ "ablehnend, sich einmischend, zustimmend oder auch fragend teil[zu]nehmen - und auf diese Weise die Tradition 
dieser Texte selber fort[zu]schreiben“ (139). Zu diesem Zwecke werden Texte zur Schöpfungserzählung (Gen 1,1-2,3; Sure 78), Opfererzählung (Gen 22,1-19), zur Berufung Mose und Auszug aus Ägypten (Ex 1,1-4,30), zum Gleichnis vom Unkraut unter dem Weizen (Mt 13,24-30), zur Nächstenliebe (Mt 5,17-20; 38-48) sowie zur Geschichte von Jesus und der Ehebrecherin (Joh 7,5- 8,11) herangezogen.

In einem ersten Schritt folgt eine exegetische Erläuterung des behandelten Textes auf der Grundlage bestimmter hermeneutischer Fragestellungen (linguistische, historisch-kontextuelle Verortung, Intention des Verfassers etc.), die für das Textverständnis relevant wären (139-144). Nachdem ein konkretes Verständnis des Textes bestimmt wurde, wird eine Hypothese darüber aufgestellt, „welche Existenzfragen im Text ausgesprochen oder bearbeitet werden“ (144). Dieser Vorgang soll u. a. eine „Auseinandersetzungs- und Verständigungsbasis“ (144) für die heutigen Menschen bzw. Schüler*innen im Unterricht liefern, auf der sie „sich mit Hilfe ihrer eigenen Lebenserfahrungen kompetent äußern können“ (144). Als didaktische Hilfestellung für die Textarbeit in der Klasse werden anschließend einige Fragen formuliert. Schließlich folgt zu jedem Text eine Erzählung, die einen möglichen Erfahrungsbezug abbildet. Allerdings ist der Zusammenhang zwischen der Erfahrungserzählung und dem behandelten Text nicht immer eindeutig erkennbar, was auch der Autor selbst in seinem Schlusswort anmerkt. Zudem stellt sich aus didaktischer Hinsicht die Frage, ob durch die Vorgabe eines (Muster)Erfahrungstextes im Grunde genommen die Erfahrungsvorstellung(en) von Schüler*innen im Hinblick auf den behandelten religiösen Text nicht prädestiniert werden, was zur Konsequenz hätte, dass der beabsichtigte individuelle Erfahrungsbezug nicht vollständig zustande kommen würde. Ferner wäre für die Umsetzung der vom Autor bearbeiteten schriftdidaktischen Unterrichtsentwürfe noch eine zusätzliche Adaptierung für den jeweiligen Unterrichtskontext bzw. der Klassenstufe erforderlich.

Aus der Perspektive einer islamischen Religionspädagogin finde ich die eigenständige exegetische Bemühung des Autors, den koranischen Text für den (christlichen) Religionsunterricht fruchtbar zu machen genauso wie seine Haltung, die religiösen Traditionen sowie die heiligen Texte "nicht von ihren Unterschieden her zu verstehen, sondern von ihren Gemeinsamkeiten" (5), beachtlich und bedeutsam. Allerdings bringt die Exegese von Schriften anderer Religionen aus der Außenperspektive die Frage mit sich, ob und inwiefern diese mit dem Selbstverständnis der Anhänger dieser Religion einhergeht - dies gilt sowohl für die Auslegung des koranischen Textes als auch der Texte aus der Tora und ihre Identifizierung mit dem jüdischen Selbstverständnis. Methodisch hilfreich wäre in diesem Fall die Einbeziehung von islamischen bzw. jüdischen Sekundärtexten, der Exegese-Literatur, und/oder der islamischen/jüdischen Expertise. Die methodische Frage - Binnen-/Außenperspektive - begegnet uns teilweise auch bei der vergleichenden Darstellung von Themen im ersten Teil, z. B. beim Koranverständnis oder beim Messianismus.

Unabhängig von diesen Fragen liefert der Beitrag wichtige Impulse für den aktuellen religionspädagogischen Gesamtdiskurs, vor allem im Hinblick auf die Frage nach den Methoden zur dialogischen und sinnstiftenden Öffnung von heiligen Texten für die gegenwärtigen Menschen, was nach wie vor sehr aktuell ist. 